\title{
EVII controls proliferation in acute myeloid leukaemia through modulation of miR-1-2
}

\author{
M Gómez-Benito' ${ }^{1,3}$, A Conchillo',3, MA García', I Vázquez ${ }^{1,2}$, M Maicas ${ }^{1,2}$, C Vicente ${ }^{1,2}$, I Cristobal 1,2, \\ N Marcotegui ', L García-Ortí', E Bandrés', MJ Calasanz ${ }^{2}$, MM Alonso*,I and MD Odero*,1,2 \\ 'Division of Oncology, Center for Applied Medical Research, University of Navarra, Navarra, Pamplona 31008, Spain; ${ }^{2}$ Department of Genetics, \\ University of Navarra, Navarra, Pamplona, Spain
}

BAKGROUND: The EVII (ecotropic virus integration site I) gene codes for a zinc-finger transcription factor, whose transcriptional activation leads to a particularly aggressive form of acute myeloid leukaemia (AML). Although, EVII interactions with key proteins in hematopoiesis have been previously described, the precise role of this transcription factor in promoting leukaemic transformation is not completely understood. Recent works have identified specific microRNA (miRNA) signatures in different AML subgroups. However, there is no analysis of miRNAs profiles associated with EVII overexpression in humans.

METHODS: We performed QT-RT-PCR to assess the expression of 250 miRNAs in cell lines with or without EVII overexpression and in patient samples. We used ChIP assays to evaluated the possible binding of EVII binding to the putative miRNA promoter. Proliferation of the different cell lines transfected with the anti- or pre-miRs was quantified by MTT.

RESULTS: Our data showed that EVII expression was significantly correlated with the expression of miR-I-2 and miR-I33-a-I in established cell lines and in patient samples. ChIP assays confirmed that EVII binds directly to the promoter of these two miRNAs. However, only miR-I-2 was involved in abnormal proliferation in EVII expressing cell lines.

CONCLUSIONS: Our data showed that EVII controls proliferation in AML through modulation of miR-I-2. This study contributes to further understand the transcriptional networks involving transcription factors and miRNAs in AML.

British Journal of Cancer (2010) I 03, 1292-1296. doi: I0.1038/sj.bjc.6605874 www.bjcancer.com

Published online 14 September 2010

(C) 2010 Cancer Research UK

Keywords: AML; EVII; MiR-I-2; MiR-I33a-I

The human EVI1 (ecotropic virus integration site 1) gene is located on 3 q26.2, a region frequently rearranged in acute myeloid leukaemia (AML) (Wieser, 2007). Most patients with 3q26 rearrangements overexpress EVI1. However, its expression is also elevated in about $10 \%$ of AML patients with no $3 \mathrm{q}$ aberrations, as a result of so far unknown mechanisms (Wieser, 2007). Of importance, EVI1 overexpression associates with poor prognosis and a shorter survival in AML (Nucifora et al, 2006).

MicroRNAs (miRNAs) are small non-coding RNAs that negatively regulate gene expression by repressing translation or inducing sequence-specific degradation of target mRNAs (Bartel, 2004). MicroRNAs play important roles in numerous cellular processes including hematopoiesis (Shivdasani, 2006; Lawrie, 2007; Fatica, 2006 no. 13). Over the past 3 years, several groups have described miRNA signatures associated with recurrent cytogenetic, molecular aberrations and with outcome in AML (Fabbri et al, 2008). However, anything is known about a miRNA profile in human AML patients with $3 \mathrm{q} 26$ rearrangements or EVI1 over-

\footnotetext{
* Correspondence: Dr MM Alonso or MD Odero;

E-mail:mmalonso@unav.es or mdodero@unav.es

${ }^{3}$ These authors equally contributed to this work

Received 30 June 2010; revised 2 August 2010; accepted 2 August 20 I0; published online 14 September 2010
}

expression. It has been reported that miRNA regulation is mediated by lineage-specific transcription factors involved in the developmental and differentiation processes (Bartel, 2004; Choong et al, 2007; Fazi et al, 2005). Interestingly, EVI1 and miRNAs have important roles in development and cell differentiation (Perkins et al, 1991). In this study, we establish a miRNA signature in AML that could be possibly regulated by overexpression of EVI1. We found a strong positive correlation between the expression levels of EVI1 and clustered microRNAs miR-1-2/ 133a-1 in AML cell lines and in patient samples. We demonstrated that EVI1 binds to a region upstream of the miRNA cluster resulting in an upregulation of $m i R-1-2 / 133 a-1$. Furthermore, functional studies showed that expression of $m i R-1-2$ was associated with enhanced proliferation in AML.

\section{MATERIALS AND METHODS}

\section{Cell lines and patient samples}

Cell lines MUTZ-3, TF-1, F-36P, HEL, HL-60, NOMO-1, MOLM-13, and OCI-AML2 were maintained in RPMI-1640, supplemented with $1 \%$ penicillin-streptomycin, and $10 \%$ FBS (GIBCO-BRL, Grand Island, NY, USA); $10 \mathrm{ng} \mathrm{ml}^{-1}$ GM-CSF was added in MUTZ-3, TF-1, and F-36P. P19 cell line was maintained in $\alpha$ MEM, 
supplemented wit $10 \%$ FBS. Patient samples were collected from the Department of Genetics, University of Navarra, Spain (Supplementary Table 1). Leukaemic blasts were obtained from bone marrow of AML patients with more than $60 \%$ blasts. The study was carried out in accordance with the ethical guidelines of our institution.

\section{Quantitative RT-PCR (qRT - PCR) of mRNA and miRNA levels}

Quantitative RT-PCR was carried out with ABI Prism-7500 (Applied Biosystems, Foster City, CA, USA) with $20 \mathrm{ng}$ of cDNA using TaqMan gene expression assays for EVI1 (Hs01118675_m1) and human GAPDH (Hs99999905_m1). Overexpression of EVI1 transcript was defined as levels higher than the average of seven bone marrow samples from healthy volunteers and three times the standard deviation. For miRNA quantification, qRT-PCR was performed with $10 \mathrm{ng}$ of total RNA using either the TaqMan miRNA Human Panel Assay Set (ED000298) or TaqMan miRNA individual assays for miR-1-2 (002222), miR-133a-1 (002246), miR146b (001097), miR-155 (002623), miR-323 (000538), miR-379 (000568) and snRNA U6B (Applied Biosystems).

\section{Western blot analysis}

Nuclear or cytoplasmatic protein samples were resolved by SDS-PAGE, electroblotted to a nitrocellulose membrane (BioRad, Hercules, CA, USA) and incubated with the appropriate antibodies: anti-EVI1 (no. 2265, Cell Signaling), anti-lamA/C (no. 2032, Cell Signaling) and anti- $\beta$-tubulin (T4026, Sigma-Aldrich, St Louis, MO, USA).

\section{Chromatin immunoprecipitation assay}

$10 \times 10^{6}$ HEL cells were used with ChIP assay kit (Upstate, Syracuse, NY, USA) according to the manufacturer instructions. Protein-DNA complexes were immunoprecipitated overnight with $5 \mu \mathrm{g}$ of anti-EVI1 (C-20) mouse antibody (sc-8707-X, Santa Cruz Biotechnology, Santa Cruz, CA, USA). Genomic regions containing the EVI1-binding sites in the pre-miR-1-2 or pre-miR-133a-1 upstream regions (Ensembl 52 miRNA genes: ENSG00000207694 and ENSG00000207786) were amplified by PCR using specific primers flanking the EVI1 binding sites: oligo no. 1 (forward), $5^{\prime}$-aaacccaggtgctcacagac- $3^{\prime}$; oligo no. 1 (reverse), 5'-cattccatagca ttgtatgttca-3'; oligo no. 2 (forward), $5^{\prime}$-ttggcaatctgtacccaaa- $3^{\prime}$; oligo no. 2 (reverse), $5^{\prime}$-tttcctgcgcttaatggttt- $3^{\prime}$. Quantification of coimmunoprecipitated promoter fragments was performed in triplicate using the SYBR-Green dye detection with oligos no. 1.

All-trans retinoic acid (ATRA) and DMSO treatments All-trans retinoic acid (ATRA) and DMSO (Sigma-Aldrich) were used as previously reported (Kazama et al, 1999). Cell morphology was evaluated in conventional light-field microscopy.

Transfection assays P19 cells were transfected with siGENOME siRNA D-006530-06 (Dharmacon, Lafayette, CO, USA) by Lipofectamine 2000 (Invitrogen, Carlsbad, CA, USA) $48 \mathrm{~h}$ after the ATRA treatment; RNA was extracted $48 \mathrm{~h}$ after transfection. For miRNA functional assays, HEL or HL-60 cells were nucleofected (Amaxa technology, Gaithersburg, MD, USA) with pre-miR-1-2 precursor (PM10617), pre-miR-1-2 inhibitor (AM10617), pre-miR133a-1 precursor (PM10413), pre-miR-133a-1 inhibitor (AM10413), pre-miRNA, or anti-miRNA scrambled control (Applied Biosystems).

\section{Statistical analysis}

Class comparison and significant analysis of microarrays (SAMs) were performed to identify differentially expressed miRNAs.
To make the analysis more restrictive and avoid false positives, $P<0.01$ was interpreted to denote statistical significance. To evaluate the correlation between EVI1 and miRNAs levels expression in cell lines and patient samples we used Spearman's rank correlation coefficient because EVI1 values lacked a normal distribution. $\triangle \mathrm{Ct}$ EVI1 (Ct EVI1-Ct GADPH) and $\triangle \mathrm{Ct}$ miRNA (Ct miRNA-Ct snRNA U6B) were used instead of raw data $\mathrm{Ct}$ as a way to normalise data. A value of $P<0.05$ was interpreted to denote statistical significance. Statistical analysis was performed using the SPSS 15.0 statistical package.

\section{RESULTS}

To identify EVI1-miRNA signatures in AML, we choose eight cell lines previously reported to be either positive or negative for EVI1 expression. We confirmed EVI1 expression at the mRNA and protein level in these cell lines (Figure $1 \mathrm{~A}$ and $\mathrm{B}$ ) and we analysed the expression levels of 250 mature miRNAs by qRT-PCR. After raw data $\mathrm{Ct}$ normalisation, statistical analysis was performed by class comparison and SAM to identify differentially expressed miRNAs between the two groups. Several miRNAs were identified by using each method, and six of them were common to both lists (Figure 1C). Among these miRNAs, we found that miR-1-2 and miR-133a-1 showed the highest correlation coefficient with EVI1 expression ( $R=0.905$ and $R=0.902$, respectively) and the best significance value $(P=0.002)$ (Figure $1 D)$. Thus, we decided to validate these two candidates in a cohort of 44 AML patient samples (20 with EVI1 overexpression). We found a significant correlation between the EVI1 mRNA and both miRNAs expression levels $(R=0.772$ and 0.695 for miR-1-2 and miR-133a-1, respectively; $P<0.001$ ) (Figure $1 \mathrm{E}$ ). Thus, EVI1 may be involved in triggering or maintaining miR-1-2 and miR-133a-1 expression in AML and favouring the perpetuation of the neoplastic phenotype in these tumours.

miR-1-2 and $m i R-133 a-1$ are clustered together in the same loci at chromosome 18 (Liu et al, 2007), suggesting that their transcription might be regulated by similar mechanisms. To elucidate whether EVI1 acts as a transcription factor for these two microRNAs, we examine their putative promoters using bioinformatics prediction tools. Interestingly, there were several binding sites for EVI1 in the upstream regions of both $m i R-1-2$ and miR-133a-1 (Figure 2A). ChIP assays showed that EVI1 was bound only to the region upstream miR-1-2, but not upstream miR-133a-1 (Figure 2B). The occupancy of this site by EVI1 was also confirmed by qRT - PCR (Figure 2C).

The P19 cell line expresses EVI1 during ATRA-induced neuroectodermal differentiation, but not during DMSO-induced mesodermal. Thus, we assess whether EVI1 induction in this model could also lead to miR-1-2/133a-1 expression. Consistently, we could detect an increase in both EVI1 and miR-1-2/133a-1 levels during ATRA-induced differentiation (Figure 2D). Importantly, reducing the levels of EVI1 by siRNA resulted concomitant decrease of these miRNAs (Figure 2E). Altogether, these data suggest a positive regulation of $m i R-1-2$ and $m i R-133 a-1$ expression by EVI1.

Finally, we wanted to elucidate whether modulation of expression levels of these two miRNAs would have a functional outcome in AML cell lines. As EVI1 overexpression has been linked with abnormal proliferation (Yuasa et al, 2005; Goyama et al, 2008) first, we assessed this function. We found that inhibition of miR-1-2 in the EVI1-positive HEL cell line induced a significant decreased in the proliferation potential of these cells. Interestingly, inhibition of miR-133a-1 resulted in a moderately decreased in the proliferation. When we transfected both of them together, we observed an effect that was very similar to the one produced by the antimiR-1 alone (Figure 2F). Of importance transfer of premiR-1, 

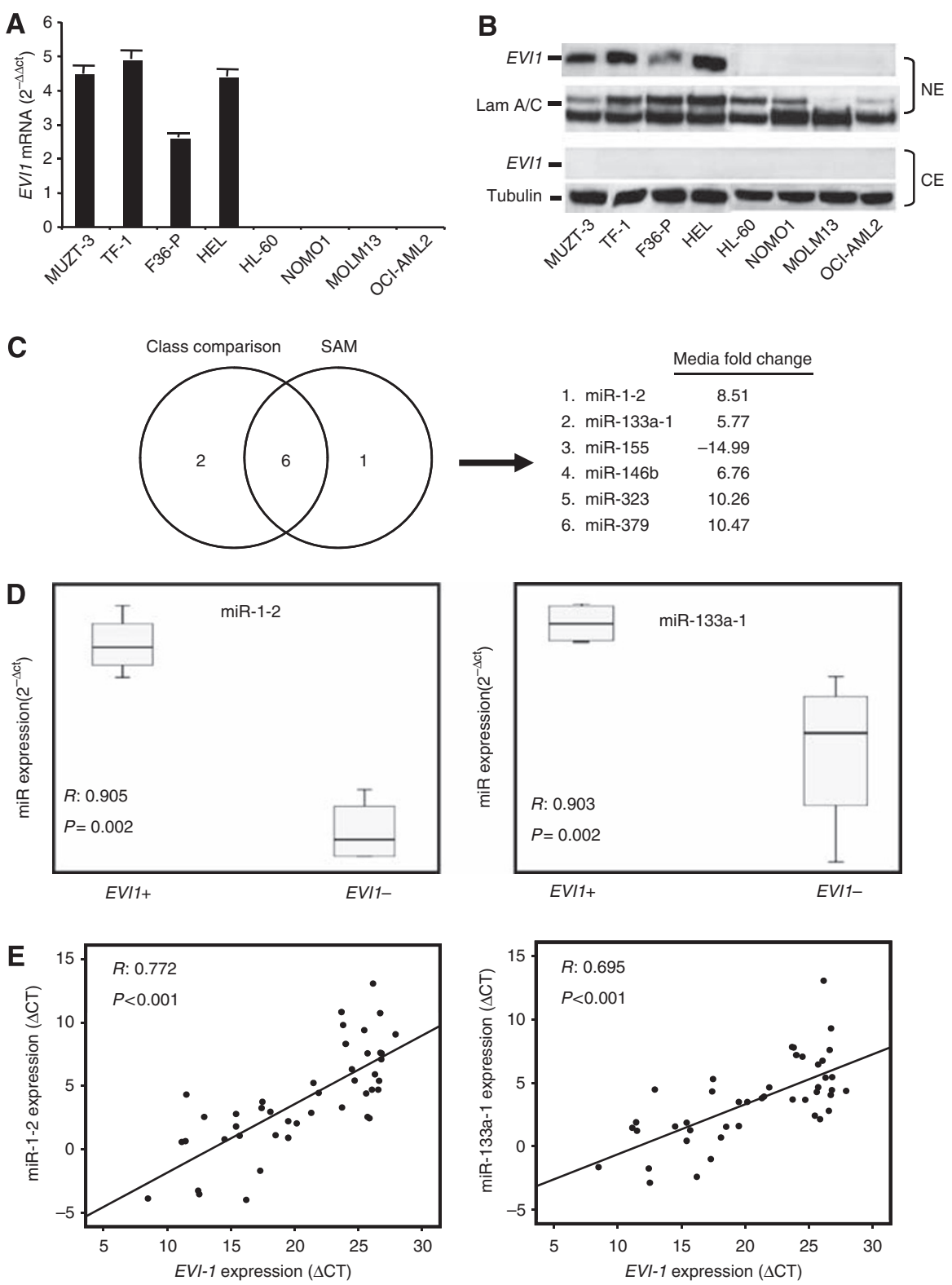

Figure I EVII and miRNAs expression in AML. (A) Relative qRT-PCR quantification of EVII transcript expression in AML cell lines. The GADPH levels were used as a normaliser for the calculation of the $2^{-\Delta \Delta C t}$ coefficient. (B) Immunoblot analysis of EVII protein levels in nuclear (NE) and cytosolic fractions (CE). Lam A/C and tubulin levels serve as control for equal protein loading. (C) Identification of miRNAs differentially expressed $(P<0.0 \mathrm{I})$ between EVII + and EVII - cell lines by class comparison and significant analysis of microarrays (SAM) methods. (D) Statistical analysis of miR-I -2 and miR-I33a-I expression levels in EVII + and EVII - cell lines. (E) Spearman's rank correlation coefficient between $\Delta \mathrm{Ct} E V I I$ and $\Delta \mathrm{Ct}$ miR-I-2 or miR-I 33a-I in patient samples.

in the EVI1-negative cell line HL-60 resulted in a significant increased in the proliferation. However, transfer of premiR-133a-1 did not show any effect (Figure 2F). These data indicated that miR-1 could be a critical regulator of proliferation in EVI1 overexpressing cell lines.

\section{DISCUSSION}

MicroRNAs have important roles in hematopoiesis, and also in the molecular pathogenesis of AML (Fabbri et al, 2008). To date, different miRNA signatures have been associated with defined AML cytogenetic subgroups. However, even though there is one study about miRNAs expression in relation with EVII overexpresssion in mice (Dickstein et al, 2010), there are no studies analysing the differential miRNAs expression in AML patients with EVII aberrant expression. Thus, this is the first study describing a miRNA profile associated with EVI1 overexpression in human AML. This EVI1-associated signature consists of six different miRNAs, of which miR-1 and miR-133a-1 showed the highest correlation with EVI1 levels in both cell lines and patient samples. Interestingly, Marcucci et al (2010) reported the upregulation of these two miRNAs in a set of AML patient with normal karyotype and a mutation in IDH2, indicating a possible role for these miRNAs in the malignant phenotype of AML patients. We found that miR-1-2 expression was involved in proliferation but not miR-133a-1. Nevertheless, we do not discard the hypothesis that miR-133a-1 could be involved in other functions such as inhibition of differentiation. 


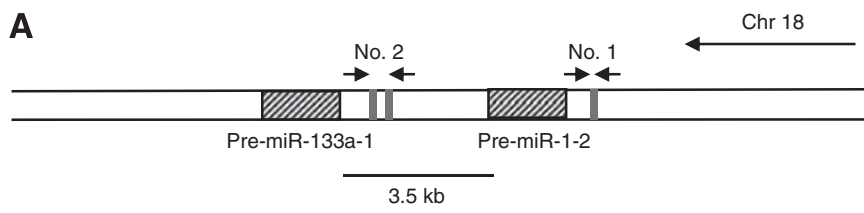

B

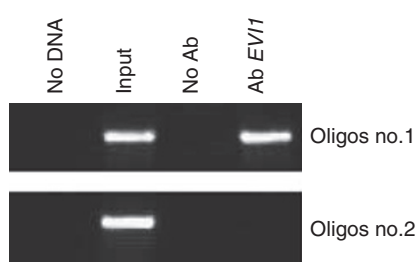

C No-Ab

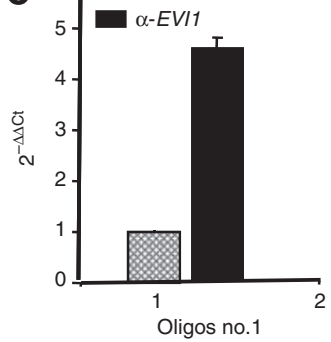

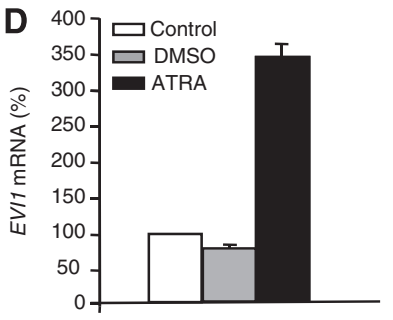

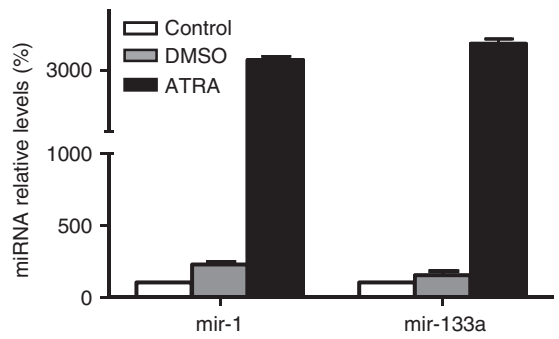

E
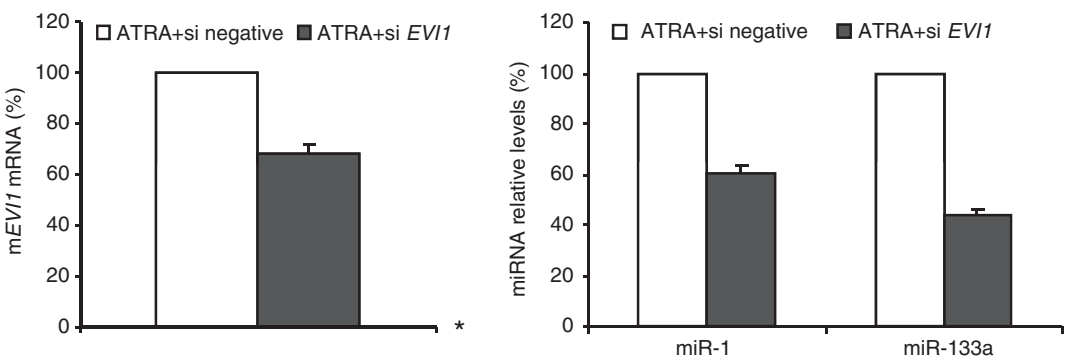

$\mathbf{F}$ HEL
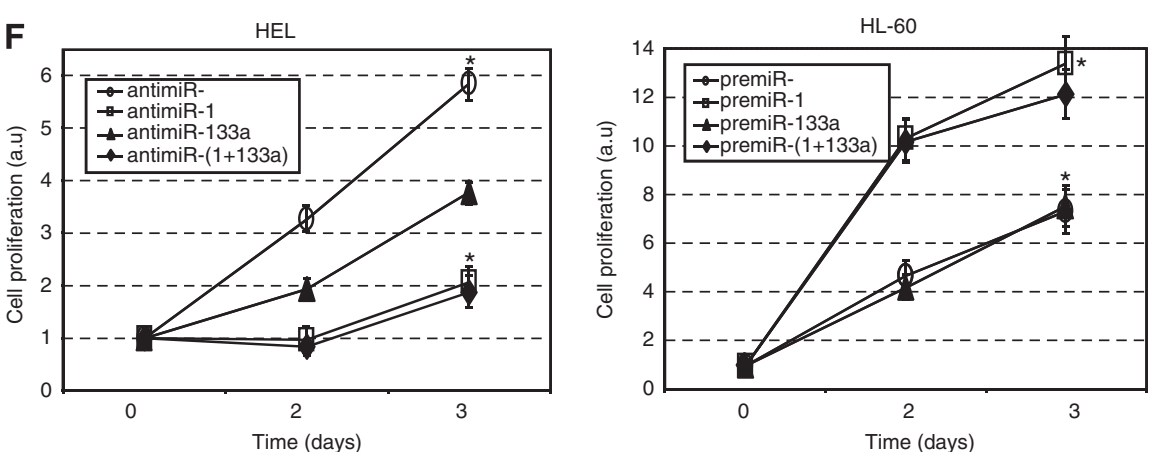

Figure $2 \mathrm{EVII}$ controls proliferation through miR-I-2 modulation. (A) Schematic representation of EVII-binding sites and PCR design in the putative promoter of miR-I-2 and miR-I33a-I. (B) The PCR amplification of EVII-immunoprecipitated DNA with the oligos no. I and oligos no. 2. Amplification of negative control (no DNA), input chromatin (input) and mock-immunoprecipitated chromatin (no Ab) were carried out as controls.

(C) Quantitative real-time PCR was performed with oligos no. I. Input chromatin was used as a normaliser. Error bars represent standard deviation. Results from a representative experiment, out of three, are shown. (D) Expression levels of mRNA EVII, miR-I-2 and miR-I33a-I in cells treated with DMSO $1 \%$ or ATRA I $\mu \mathrm{M}$ relative to PI9 control cells, $\beta$-actin and snRNA U6B levels were used as normalisers for mRNA and miRNAs levels, respectively. (E) Quantification of mRNA EVII, miR-I-2, and miR-/33a-I in PI9 transfected with a scramble or an EVII siRNA, during the ATRA treatment. (F) HEL and HL-60 cells were respectively transfected with premiRs or anti-miRs, as indicated, and cell proliferation was measured at different time points. Untreated cells day 0 was given an arbitrary value of $I$ and all the values were normalised with this group. Abbreviation: a.u. $=$ arbitrary unit.

In conclusion, this study provides new insight in our understanding of the role of EVI1 in AML. Our results demonstrate for the first time a functional relationship between EVI1 and miRNAs expression. Moreover, uncovers a new pathway that could lead to the development of novel therapeutic approaches to treat EVI1-overexpressing leukaemia patients. 


\section{ACKNOWLEDGEMENTS}

This work was supported by Ministerio de Salud (FIS-CD07/ 00045), Ministerio de Educación y Ciencia (SAF2005/06425), Ministerio de Ciencia e Innovación (PI081687), ISCIII-RETIC (RD06/0020/0078 and RD06/0020/0071), Departamento Salud del

\section{REFERENCES}

Bartel DP (2004) MicroRNAs: genomics, biogenesis, mechanism, and function. Cell 116: $281-297$

Choong ML, Yang HH, McNiece I (2007) MicroRNA expression profiling during human cord blood-derived CD34 cell erythropoiesis. Exp Hematol 35: $551-564$

Dickstein J, Senyuk V, Premanand K, Laricchia-Robbio L, Xu P, Cattaneo F, Fazzina R, Nucifora G (2010) Methylation and silencing of miRNA-124 by EVI1 and self-renewal exhaustion of hematopoietic stem cells in murine myelodysplastic syndrome. Proc Natl Acad Sci USA 107: $9783-9788$

Fabbri M, Garzon R, Andreeff M, Kantarjian HM, Garcia-Manero G, Calin GA (2008) MicroRNAs and noncoding RNAs in hematological malignancies: molecular, clinical and therapeutic implications. Leukemia 22: 1095 - 1105

Fatica A, Rosa A, Fazi F, Ballarino M, Morlando M, De Angelis FG, Caffarelli E, Nervi C, Bozzoni I (2006) MicroRNAs and hematopoietic differentiation. Cold Spring Harb Symp Quant Biol 71: 205-210

Fazi F, Rosa A, Fatica A, Gelmetti V, De Marchis ML, Nervi C, Bozzoni I (2005) A minicircuitry comprised of microRNA-223 and transcription factors NFI-A and C/EBP alpha regulates human granulopoiesis. Cell 123: $819-831$

Goyama S, Yamamoto G, Shimabe M, Sato T, Ichikawa M, Ogawa S, Chiba S, Kurokawa M (2008) Evi-1 is a critical regulator for hematopoietic stem cells and transformed leukemic cells. Cell Stem Cell 3: 207-220

Kazama H, Kodera T, Shimizu S, Mizoguchi H, Morishita K (1999) Ecotropic viral integration site- 1 is activated during, and is Sufficient for, neuroectodermal P19 cell differentiation. Cell Growth Diff 10: 565-573
Gobierno de Navarra (14/2008), and Fundación para la Investigación Médica Aplicada y UTE (Spain).

Supplementary Information accompanies the paper on British Journal of Cancer website (http://www.nature.com/bjc)

Lawrie CH (2007) MicroRNAs and haematology: small molecules, big function. Br J Haematol 137: 503-512

Liu N, Williams AH, Kim Y, McAnally J, Bezprozvannaya S, Sutherland LB, Richardson JA, Bassel-Duby R, Olson EN (2007) An intragenic MEF2dependent enhancer directs muscle-specific expression of microRNAs 1 and 133. Proc Natl Acad Sci USA 104: 20844-20849

Marcucci G, Maharry K, Wu YZ, Radmacher MD, Mrozek K, Margeson D, Holland KB, Whitman SP, Becker H, Schwind S, Metzeler KH, Powell BL, Carter TH, Kolitz JE, Wetzler M, Carroll AJ, Baer MR, Caligiuri MA, Larson RA, Bloomfield CD (2010) IDH1 and IDH2 gene mutations identify novel molecular subsets within de novo cytogenetically normal acute myeloid leukemia: a Cancer and Leukemia Group B study. J Clin Oncol 28: $2348-2355$

Nucifora G, Laricchia-Robbio L, Senyuk V (2006) EVI1 and hematopoietic disorders: history and perspectives. Gene 368: $1-11$

Perkins AS, Mercer JA, Jenkins NA, Copeland NG (1991) Patterns of Evi-1 expression in embryonic and adult tissues suggest that Evi-1 plays an important regulatory role in mouse development. Development 111: $479-487$

Shivdasani RA (2006) MicroRNAs: regulators of gene expression and cell differentiation. Blood 108: 3646-3653

Wieser R (2007) The oncogene and developmental regulator EVI1: expression, biochemical properties, and biological functions. Gene 396: $346-357$

Yuasa H, Oike Y, Iwama A, Nishikata I, Sugiyama D, Perkins A, Mucenski ML, Suda T, Morishita K (2005) Oncogenic transcription factor Evil regulates hematopoietic stem cell proliferation through GATA-2 expression. EMBO J 24: $1976-1987$ 